PROCEEDINGS OF THE

AMERICAN MATHEMATICAL SOCIETY

Volume 140, Number 8, August 2012, Pages 2767-2782

S 0002-9939(2011)11122-5

Article electronically published on December 7, 2011

\title{
IMPLICIT DIFFERENTIAL INCLUSIONS IN REFLEXIVE SMOOTH BANACH SPACES
}

\author{
MESSAOUD BOUNKHEL
}

(Communicated by Yingfei Yi)

\begin{abstract}
In this paper we prove the existence of solutions of some types of implicit differential inclusions in reflexive smooth Banach spaces. A positive answer is given to a question of Ding [On a class of implicit differential inclusions, Proc. Amer. Math. Soc., 124 (1996), no. 3, 745-749.]
\end{abstract}

\section{INTRODUCTION}

We are given a real interval $I=[0, T](T>0)$ and consider the initial value problem of the form

$$
0 \in G(t, u(t), \dot{u}(t)), \text { a.e. on } I, u(0)=x_{0},
$$

where $G$ is a set-valued mapping defined from $I \times \mathbb{R}^{n} \times \mathbb{R}^{n}$ to $\mathbb{R}^{n}$. This type of problem is called Implicit Differential Inclusions (IDI). Taking $F$, a single-valued mapping, we obtain the well known implicit differential equations. There are many works that deal in the theory of implicit differential equations (see for instance [11, 21]). In the two last decades more attention has been given to the set-valued case with some particular forms of $G$. Let us state some of them in chronological order.

In 4, the authors proved the existence of solutions for (IDI) with $G(t, x, y)=$ $-f(t, x, y)+F(t, x)$, where $f$ is a continuous single-valued mapping and $F$ is upper semi-continuous with closed convex images in $\mathbb{R}^{n}$. Their idea is to rewrite (IDI) in an equivalent way as an explicit differential inclusion (DI) and then they use the existence results for (DI) to get solutions for the corresponding (IDI). Using the same ideas, Wenzel studied in 29] the case when $G$ has the form $G(t, x, y)=M(y)+C(x)$, where $C$ and $M$ are two maximal monotone operators with images in $\mathbb{R}^{n}$. Later on, Ding extends in [30] the existence result of [29] from the case in which $C$ is a maximal monotone operator to the case where $C$ is an u.s.c. set-valued mapping with compact convex values. The proof in [30] is based on an approximation approach. Recently, Kuttler in [19] studied in the case of separable reflexive Banach spaces $X$ the problem (IDI) with $G(t, u(t), \dot{u}(t))=-f(t)+A(t, u(t))+(\widehat{(\overrightarrow{B(t) u}(t)})$, where $A$ is monotone, $B$ is linear with some additional assumptions on $A, B$, and $f$. Two other types of (IDI) have been the subject of two papers by Manchanda and Siddiqi [22, 27] in the case where $X=H$ is a Hilbert space. These two types

Received by the editors November 9, 2009 and, in revised form, March 6, 2010 and March 7, 2010 .

2010 Mathematics Subject Classification. Primary 34A60, 49J53.

Key words and phrases. Uniformly smooth and uniformly convex Banach spaces, implicit convex sweeping process, generalized projection, duality mapping.

(C)2011 American Mathematical Society 
correspond to the form $G(t, x, y)=x+F(t, y)$ and $G(t, x, y)=y+F(t, y)$, where $F(t, y)$ has the special form $F(t, y)=N(C(t), y)$ which denotes the normal cone at $y \in C(t)$ to some closed convex moving set $C(t) \subset H$. The proofs for these two last forms are completely different from the previous ones. They are based on the construction of sequences of mappings using the projection operator. It uses some different techniques and concepts which have been used to solve a particular type of differential inclusion, called sweeping processes (for more details on these problems and their applications, we refer the reader to [5, 6, 7, 22, 27]). The case $G(t, y)=x+N(C(t), y)$ has been extended by the author in [7] from the convex case of $C(t)$ to the nonconvex case in Hilbert spaces.

The aim of the present paper is to continue the study of the existence of solutions for (IDI) when $G$ has the general form $G(t, x, y)=N(C(t), y)+F(t, x)+y$ and when the space $X$ is a separable reflexive Banach space. The paper is organized as follows. Section 2 contains some definitions, notations, and important results needed in the paper. In Section 3, we state and prove our main results.

\section{Preliminaries}

In the sequel, $X$ is a Banach space with topological dual space $X^{*}$. We denote by $d_{S}$ the usual distance function to $S$, i.e., $d_{S}(x):=\inf _{u \in S}\|x-u\|$. We need first to recall some notation and definitions needed in the paper. Let $S$ be a nonempty closed convex set of $X$ and let $\bar{x}$ be a point in $S$. The convex normal cone of $S$ at $\bar{x}$ is defined by (see for instance [13])

$$
N(S ; \bar{x})=\left\{\varphi \in X^{*}:\langle\varphi, x-\bar{x}\rangle \leq 0 \text { for all } x \in S\right\} .
$$

It is well known (see for example [14]) that $N(S ; \bar{x})$, the normal cone of a closed convex set $S$ at $\bar{x} \in S$, can be defined, when $X$ is Hilbert, in terms of the projection operator $P_{S}$ as follows:

$$
N(S ; \bar{x})=\left\{\xi \in X^{*}: \exists \alpha>0 \text { so that } \bar{x} \in P_{S}(\bar{x}+\alpha \xi)\right\} .
$$

A Banach space $(X,\|\cdot\|)$ is said to be smooth provided the limit

$$
\lim _{t \rightarrow 0} \frac{\|x+t y\|-\|x\|}{t}
$$

exists for each $x, y \in X$ satisfying $\|x\|=\|y\|=1$. In this case, the norm of $X$ is said to be Gâteaux differentiable. The normalized duality mapping $J: X \rightrightarrows X^{*}$ is defined by

$$
J(x)=\left\{j(x) \in X^{*}:\langle j(x), x\rangle=\|j(x)\|\|x\|=\|x\|^{2}=\|j(x)\|^{2}\right\} .
$$

Many properties of the normalized duality mapping $J$ have been studied. For the details, one may see the books [1, 28, 31].

Let $V: X^{*} \times X \rightarrow \mathbb{R}$ be defined by

$$
V(\varphi, x)=\|\varphi\|^{2}-2\langle\varphi, x\rangle+\|x\|^{2}, \text { for any } \varphi \in X^{*} \text { and } x \in X .
$$

We list some important properties of $V$ needed in our proofs when $X$ is a reflexive smooth Banach space:

i) $V(\varphi, x) \geq 0, \forall x \in X, \forall \varphi \in X^{*}$;

ii) $(\|\varphi\|-\|x\|)^{2} \leq V(\varphi, x) \leq(\|\varphi\|+\|x\|)^{2}, \forall x \in X, \forall \varphi \in X^{*}$;

iii) $V(J(x), x)=0, \forall x \in X$. 
Based on the functional $V$, a set $\pi_{S}(\varphi)$ of generalized projections of $\varphi \in X^{*}$ onto $S$ is defined as follows (see [2, 3]).

Definition 2.1. Let $S$ be a nonempty subset of $X$ and $\varphi \in X^{*}$. If there exists a point $\bar{x} \in S$ satisfying

$$
V(\varphi, \bar{x})=\inf _{x \in S} V(\varphi, x),
$$

then $\bar{x}$ is called a generalized projection of $\varphi$ onto $S$. The set of all such points is denoted by $\pi_{S}(\varphi)$. When the space $X$ is not reflexive, $\pi_{S}(\varphi)$ may be empty for some elements $\varphi \in X^{*}$ even when $S$ is closed and convex (see Example 1.4. in [20]).

The following theorem summarizes some important properties of the set $\pi_{S}(\varphi)$ needed in our proofs. For the proof of these results we refer the reader to [1, 2, 3].

Theorem 2.1. Let $X$ be a reflexive Banach space with dual space $X^{*}$ and let $S$ be a nonempty, closed and convex subset of $X$. The following properties hold:

$\left(\pi_{1}\right) \pi_{S}(\varphi) \neq \emptyset$, for any $\varphi \in X^{*}$;

$\left(\pi_{2}\right)$ If $X$ is also smooth, then for any given $\varphi \in X^{*}, \bar{x} \in \pi_{S}(\varphi)$ if and only if $\langle\varphi-J(\bar{x}), x-\bar{x}\rangle \leq 0$ for all $x \in S$

$\left(\pi_{3}\right) \pi_{S}(\varphi)$ is singleton for all $\varphi \in X^{*}$ if and only if $X$ is strictly convex.

We have seen that if $X$ is a reflexive smooth Banach space, $J$ is a single valued mapping from $X$ onto $X^{*}$. Then Theorem 3.1 in [26] ensures the following result.

Theorem 2.2. For a nonempty closed convex subset $S$ of a reflexive smooth Banach space $X$ and $u \in S$, the following assertions are equivalent:

i) $\bar{x} \in S$ is a projection of $u$ onto $S$, that is $\bar{x} \in P_{S}(u)$;

ii) $\langle J(u-\bar{x}), x-\bar{x}\rangle \leq 0$ for all $x \in S$;

iii) $J(u-\bar{x}) \in N(S ; \bar{x})$.

We will use the following theorem, which establishes a new characterization of the normal cone for closed convex sets in reflexive smooth Banach spaces in terms of the generalized projection $\pi_{S}$. For its proof we refer the reader to 9 .

Theorem 2.3. Let $X$ be a reflexive smooth Banach space, let $S$ be a nonempty closed convex subset of $X$, and let $\bar{x} \in S$. The following assertions are equivalent:

i) $x^{*} \in N(S, \bar{x})$;

ii) there exists $\alpha>0$ such that $\bar{x} \in \pi_{S}\left(J(\bar{x})+\alpha x^{*}\right)$.

We end this section with the following lemma needed in our proofs (for its proof we refer the reader, for instance, to [12]).

Lemma 2.4. Let $X$ be a reflexive Banach space and let $C: I \rightarrow X$ be a set-valued mapping with nonempty closed convex values. Then the functional $I: v \mapsto I(v):=$ $\int_{0}^{T} \delta_{C(t)}^{*}(v(t)) d t$ from $X^{*}$ to $\mathbb{R}$ is weak lower semi-continuous in the following sense: for any $v_{n}$ a sequence of mappings $v_{n}: I \rightarrow X^{*}$ such that $v_{n} \rightarrow v_{*}$ in the weak star topology of $L^{\infty}\left(I, X^{*}\right)$, we have

$$
\int_{0}^{T} \delta_{C(t)}^{*}\left(v_{*}(t)\right) d t \leq \liminf _{n} \int_{0}^{T} \delta_{C(t)}^{*}\left(v_{n}(t)\right) d t .
$$




\section{MAin RESUlts}

We start this section by proving the following existence result of approximate solutions to an implicit variant of sweeping processes with a perturbation in any separable reflexive smooth Banach space.

Theorem 3.1. Let $X$ be a separable reflexive smooth Banach space, let $T>0$, $I=[0, T]$, and $C: I \rightrightarrows X$ be a set-valued mapping with closed convex values and let $F: I \times X \rightarrow X^{*}$ be a set-valued mapping with closed values satisfying

$$
F(t, x) \subset \beta B_{*}, \text { for all } t \in I, x, y \in X \text { with } \beta \geq 0 \text {. }
$$

Assume that for some positive number $R$ we have $d_{C(t)}(0)<R$ for all $t \in I$ (i.e., the distance of the origin to the values $C(t)$ is bounded by some positive number $R$ ). Then for any initial points $x_{0} \in X$, there exist sequences of mappings $\theta_{n}: I \rightarrow I$, $\rho_{n}: I \rightarrow I, \eta_{n}: I \rightarrow I, u_{n}: I \rightarrow X$ such that $\theta_{n}(t) \rightarrow t, \rho_{n}(t) \rightarrow t, \theta_{n}(t) \rightarrow t$, uniformly on $I$, and $\left\|u_{n}(t)\right\| \leq T(R+2 \beta)+\left\|x_{0}\right\|$, and $\left\|\dot{u}_{n}(t)\right\| \leq R+2 \beta$ a.e. on $I$, and the following differential inclusion holds for almost all $t$ in $I$ :

$$
-J\left(\dot{u}_{n}(t)\right) \in N\left(C\left(\theta_{n}(t)\right) ; \dot{u}_{n}(t)\right)+F\left(\rho_{n}(t), u_{n}\left(\rho_{n}(t)\right)\right),
$$

with $u_{n}(0)=x_{0}$.

Remark 3.1. Before stating the proof of this theorem we give an example of a set-valued mapping $C$ satisfying the assumptions on $C$. Take $X=\mathbb{R}^{2}, C(t)=$ $\mathbb{R}_{+}^{2}+(t, 0)$. Clearly $C$ has closed convex cone values (so not necessarily bounded values) with Lipschitz ratio $\lambda=1$, and the distance from $(0,0)$ to the values of $C$ is bounded by $T$.

Proof of Theorem 3.1. Fix $n_{0} \in \mathbb{N}$ such that $n_{0}>T$. For every $n>n_{0}$, we put $\mu_{n}:=T / n<1$, and we consider the following partition of $I$ :

$$
\begin{gathered}
t_{n, i}:=i \mu_{n}(\text { for } 0 \leq i \leq n,) \text { and } \\
\left.\left.I_{n, i+1}:=\right] t_{n, i}, t_{n, i+1}\right](\text { for } 0 \leq i \leq n-1,) \text { and } I_{n, 0}:=\left\{t_{n, 0}\right\} .
\end{gathered}
$$

For every $n>n_{0}$, we choose by induction

$$
\begin{aligned}
u_{n, 0} & =x_{0} ; \quad z_{n, 0}^{*} \in F\left(t_{n, 0}, x_{0}\right), \\
u_{n, i+1} & \in u_{n, i}+\mu_{n} \pi_{C\left(t_{n, i+1}\right)}\left(-z_{n, i}^{*}\right), \\
z_{n, i}^{*} & \in F\left(t_{n, i}, u_{n, i}\right) .
\end{aligned}
$$

This induction is well defined by using the assumption that $C(t)$ is nonempty, closed and convex for any $t \in I$ and Theorem 2.1. The sequences $\left\{u_{n, i}\right\}_{i}$ and $\left\{z_{n, i}^{*}\right\}_{i}$ are used to construct two sequences of mappings $u_{n}$ from $I$ to $X$ and $z_{n}^{*}$ from $I$ to $X^{*}$ by defining their restrictions to each interval $I_{n, i}$ as follows:

$$
\begin{gathered}
\text { for } t=0, \text { set } z_{n}^{*}(t):=z_{n, 0}^{*}, \text { and } u_{n}(t):=u_{n, 0} \\
\text { for all } t \in I_{n, i+1}(0 \leq i \leq n-1), \text { set } z_{n}^{*}(t):=z_{n, i}^{*} \text {, } \\
\text { and } u_{n}(t):=u_{n, i}+\frac{\left(t-t_{n, i}\right)}{\mu_{n}}\left(u_{n, i+1}-u_{n, i}\right)
\end{gathered}
$$

It is clear by construction that $u_{n}\left(t_{n, i}\right)=u_{n, i}$ and

$$
\left.\dot{u}_{n}(t)=\frac{u_{n, i+1}-u_{n, i}}{\mu_{n}} \in \pi_{C\left(t_{n, i+1}\right)}\left(-z_{n}^{*}(t)\right) \text {, for all } t \in\right] t_{n, i}, t_{n, i+1}[\text {. }
$$


Define now the functions $\theta_{n}$ and $\rho_{n}$ from $I$ to $I$ as follows: $\theta_{n}(t)=t_{n, i+1}$ and $\rho_{n}(t)=t_{n, i}$ for any $t \in I_{n, i+1}$. Clearly $\theta_{n}(t)$ and $\rho_{n}(t)$ converge to $t$ uniformly on I. Now, we put for any $n>n_{0}, w_{n, i+1}:=\frac{u_{n, i+1}-u_{n, i}}{\mu_{n}}$, for any $0 \leq i \leq n-1$.

Claim 1. Let us prove that the sequence of points $\left(w_{n, i}\right)_{i}$ is bounded by a positive constant. First observe that the definition of the generalized projection stated in Section 2 and the properties of the functional $V$ entail

$$
\begin{aligned}
\left\|w_{n, i}\right\| & =\sqrt{V\left(0, w_{n, i}\right)} \\
& \leq \sqrt{V\left(-z_{n, i}^{*}, w_{n, i}\right)}+\left\|z_{n, i}^{*}\right\| \\
& =\sqrt{\inf _{y \in C\left(t_{n, i+1}\right)} V\left(-z_{n, i}^{*}, y\right)}+\left\|z_{n, i}^{*}\right\| \\
& \leq \sqrt{\inf _{y \in C\left(t_{n, i+1}\right)}\left(\left\|z_{n, i}^{*}\right\|+\|y\|\right)^{2}}+\left\|z_{n, i}^{*}\right\| \\
& =\sqrt{\left(\left\|z_{n, i}^{*}\right\|+\inf _{y \in C\left(t_{n, i+1}\right)}\|y\|\right)^{2}}+\left\|z_{n, i}^{*}\right\| \\
& \leq \sqrt{\left(\left\|z_{n, i}^{*}\right\|+R\right)^{2}}+\left\|z_{n, i}^{*}\right\|=R+2\left\|z_{n, i}^{*}\right\| .
\end{aligned}
$$

By the boundedness of $F$ we have $\left\|z_{n, i}^{*}\right\| \leq \beta$, and so

$$
\left\|w_{n, i}\right\| \leq R+2 \beta \text {. }
$$

This ensures that the sequence $w_{n, i}$ is bounded. It follows from this claim and the construction of $u_{n}$ that

$$
\left\|\dot{u}_{n}(t)\right\| \leq R+2 \beta, \text { for any } t \in I \backslash\left\{t_{n, i}\right\},
$$

which ensures that

$$
\left\|u_{n}(t)\right\| \leq\left\|x_{0}\right\|+T(R+2 \beta) .
$$

Claim 2. $t \mapsto u_{n}(t)$ are equi-Lipschitz. Let us prove now that the mappings $\left\{u_{n}\right\}$ are Lipschitz with ratio $\lambda=R+2 \beta$. Let $t, s \in I$. Then $t \in I_{n, i}$ and $s \in I_{n, j}$ for some $i$ and $j$ in $\{0, n-1\}$ (without loss of generality we assume that $i \leq j$ ). Using our construction and Claim 1 we obtain

$$
\begin{aligned}
\left\|u_{n}(t)-u_{n}(s)\right\| \leq & \left\|u_{n}(t)-u_{n}\left(t_{n, j}\right)\right\|+\sum_{k=i+1}^{j-1}\left\|u_{n}\left(t_{n, k+1}\right)-u_{n}\left(t_{n, k}\right)\right\| \\
& +\left\|u_{n}\left(t_{n, i+1}\right)-u_{n}(s)\right\| \\
\leq & \left(t-t_{n, j}\right)\left\|w_{n, j+1}\right\|+\sum_{k=i+1}^{j-1}\left\|u_{n, k+1}-u_{n, k}\right\| \\
& +\left(t_{n, i+1}-s\right)\left\|w_{n, i+1}\right\| \\
& \leq\left(t-t_{n, j}\right)\left\|w_{n, j+1}\right\|+\sum_{k=i+1}^{j-1}\left(t_{n, k+1}-t_{n, k}\right)\left\|w_{n, k+1}\right\| \\
& +\left(t_{n, i+1}-s\right)\left\|w_{n, i+1}\right\| \\
& \leq \lambda\left(t-t_{n, j}\right)+\sum_{k=i+1}^{j-1} \lambda\left(t_{n, k+1}-t_{n, k}\right)+\lambda\left(t_{n, i+1}-s\right)=\lambda(t-s) .
\end{aligned}
$$


Interchanging the roles of $t$ and $s$ we get the Lipschitz continuity of $u_{n}$. To complete the proof we have to prove (3.2). Let $t \in] t_{n, i}, t_{n, i+1}[$. Then, by our construction we have

$$
\dot{u}_{n}(t) \in \pi_{C\left(t_{n, i+1}\right)}\left(-z_{n}^{*}(t)\right) \text { and } z_{n}^{*}(t) \in F\left(t_{n, i}, u_{n}\left(t_{n, i}\right)\right) .
$$

Using Theorem 2.3 we obtain

$$
\left.-J\left(\dot{u}_{n}(t)\right) \in N\left(C\left(t_{n, i+1}\right) ; \dot{u}_{n}(t)\right)+F\left(t_{n, i}, u_{n}\left(t_{n, i}\right)\right), \forall t \in\right] t_{n, i}, t_{n, i+1}[,
$$

and hence

$$
-J\left(\dot{u}_{n}(t)\right) \in N\left(C\left(\theta_{n}(t)\right) ; \dot{u}_{n}(t)\right)+F\left(\rho_{n}(t), u_{n}\left(\rho_{n}(t)\right)\right) \text {, a.e. on } I .
$$

Thus the proof is complete.

Using the previous approximate result, we are going to prove an existence result for an implicit variant of sweeping processes with a perturbation in separable Hilbert spaces. Throughout the sequel we assume that $F$ is a completely continuous mapping from $I \times X$ to $X^{*}$, that is, $\forall s_{n} \rightarrow s$ in $I$ and $\forall x_{n} \rightarrow x$ weakly in $X$, then we have $F\left(s_{n}, x_{n}\right) \rightarrow F(s, x)$ strongly in $X^{*}$. By Proposition 7.6 in [17, every compact operator is completely continuous and the inverse is not true in general (see for instance [17). As an example for completely continuous operators, we take $X:=L_{2}([0,1], \mathbb{R}), T:=1$, and define $F: I \times X \rightarrow X^{*}$ by

$$
(t, x) \mapsto F(t, x) \in L_{2}([0,1], \mathbb{R}): \tau \mapsto F(t, x)(\tau)=\int_{0}^{1} K(\tau, s)[x(s)+t s] d s,
$$

where $K \in L_{2}([0,1] \times[0,1], \mathbb{R})$. Using the ideas in [17, pp. 206-207] we can prove that $F$ is completely continuous whenever $K$ is continuous.

Theorem 3.2. Let $X=\mathbb{H}$ be a separable Hilbert space, and assume that $C$ and $F$ satisfy the assumptions in Theorem 3.1. Assume, in addition, that $F$ is a completely continuous mapping and $C$ is Hausdorff Lipschitz with ratio $k$; i.e., for any $t, t^{\prime} \in I$

$$
\mathcal{H}\left(C\left(t^{\prime}\right) ; C(t)\right)|\leq k| t^{\prime}-t \mid \text { for some constant } k>0 .
$$

Here $\mathcal{H}$ denotes the Hausdorff distance relative to the norm associated with the space $X$ defined by

$$
\mathcal{H}(A, B):=\max \left\{\sup _{a \in A} d_{B}(a), \sup _{b \in B} d_{A}(b)\right\} .
$$

Then for any initial points $x_{0} \in X$, there exists a Lipschitz continuous mapping $u: I \rightarrow H$ satisfying

$$
-\dot{u}(t) \in N(C(t) ; \dot{u}(t))+F(t, u(t)), \text { a.e. on } I,
$$

with $u(0)=x_{0}$ and

$$
\|\dot{u}(t)\| \leq R+2 \beta \text { a.e. on } I \text {, and }\|u(t)\| \leq T(R+2 \beta)+\left\|x_{0}\right\| \text { on } I .
$$

Proof. We use the fact that in Hilbert spaces $J$ is the identity operator, and by Theorem 3.1 there exist sequences of mappings $\theta_{n}, \rho_{n}: I \rightarrow I, u_{n}: I \rightarrow X$ such that $\left\|u_{n}(t)\right\| \leq T(R+2 \beta)+\left\|x_{0}\right\|$ and $\left\|\dot{u}_{n}(t)\right\| \leq R+2 \beta$ and for almost all $t$ in $I$,

$$
-\dot{u}_{n}(t) \in N\left(C\left(\theta_{n}(t) ; \dot{u}_{n}(t)\right)\right)+F\left(\rho_{n}(t), u_{n}\left(\rho_{n}(t)\right)\right),
$$

with $u_{n}(0)=x_{0}$. Using the fact that $L^{\infty}(I, H)$ is the dual space of the separable Banach space $L^{1}(I, H)$, we can extract a subsequence still denoted $\dot{u}_{n}$ which converges 
to some $v_{*}$ in the weak star topology in $L^{\infty}(I, H)$. Then, for any $\phi \in L^{1}(I, H)$, we have $\left\langle\dot{u}_{n}-v_{*}, \phi\right\rangle_{L^{\infty}, L^{1}} \rightarrow 0$, as $n \rightarrow+\infty$, that is,

$$
\left\langle\int_{0}^{t}\left[\dot{u}_{n}(s)-v_{*}(s)\right] d s, \xi\right\rangle \rightarrow 0, \forall \xi \in H .
$$

Let $u(t):=x_{0}+\int_{0}^{t} v_{*}(s) d s, \forall t \in I$. Then,

$$
\left\langle u_{n}(t)-u(t), \xi\right\rangle=\left\langle\int_{0}^{t}\left[\dot{u}_{n}(s)-v_{*}(s)\right] d s, \xi\right\rangle \rightarrow 0, \forall \xi \in H
$$

that is, $u_{n}(t)$ weakly converges in $H$ to $u(t)$ for all $t \in I$ and $v_{*}(t)=\dot{u}(t)$ a.e. on $I$. The weak convergence of $u_{n}\left(\rho_{n}(t)\right)$ to $u(t)$ in $H$, for all $t \in I$, follows from the two following facts:

$$
\left\langle u_{n}\left(\rho_{n}(t)\right)-u(t), \xi\right\rangle=\left\langle u_{n}\left(\rho_{n}(t)\right)-u_{n}(t), \xi\right\rangle+\left\langle u_{n}(t)-u(t), \xi\right\rangle, \forall \xi \in H
$$

and

$\left|\left\langle u_{n}\left(\rho_{n}(t)\right)-u_{n}(t), \xi\right\rangle\right|=\left|\left\langle\int_{t}^{\rho_{n}(t)} \dot{u}_{n}(s) d s, \xi\right\rangle\right| \leq\|\xi\|(R+2 \beta)\left|\rho_{n}(t)-t\right| \rightarrow 0, \forall \xi \in H$.

Let $z_{n}^{*}$ be defined as in the proof of Theorem 3.1 so $z_{n}^{*}(t)=F\left(\rho_{n}(t), u_{n}\left(\rho_{n}(t)\right)\right)$. Then the complete continuity of $F$ ensures for all $t \in I, z^{*}(t) \rightarrow F(t, u(t))$ strongly in $X^{*}$. By our construction and the characterization of the normal cone for closed convex sets given in Theorem 2.2, we have

$$
\left\langle-\dot{u}_{n}(t)-z_{n}^{*}(t) ; w-\dot{u}_{n}(t)\right\rangle \leq 0, \quad \text { for all } w \in C\left(\theta_{n}(t)\right) \text {, a.e. on } I .
$$

Now let any $t \in I$ for which (3.8) holds and let any $v \in C(t)$. By the Hausdorff Lipschitz property of $C$, we can write $v \in C\left(\theta_{n}(t)\right)+k\left|t-\theta_{n}(t)\right| \mathbb{B}$. Hence, there exists $w \in C\left(\theta_{n}(t)\right)$, such that $\|w-v\| \leq k\left|t-\theta_{n}(t)\right|$. Therefore, by (3.8) we can write

$$
\begin{aligned}
& \left\langle-\dot{u}_{n}(t)-z_{n}^{*}(t) ; v-\dot{u}_{n}(t)\right\rangle \\
& \quad=\left\langle-\dot{u}_{n}(t)-z_{n}^{*}(t) ; w-\dot{u}_{n}(t)\right\rangle+\left\langle-\dot{u}_{n}(t)-z_{n}^{*}(t) ; v-w\right\rangle \\
& \quad \leq\left\|\dot{u}_{n}(t)+z_{n}^{*}(t)\right\|\|v-w\|, \\
& \quad \leq \delta\|v-w\| \\
& \quad \leq \delta k \mu_{n}
\end{aligned}
$$

a.e. on $I$, with $\delta=R+3 \beta$, and so for a.e. $t \in I$ we get

$$
\left.\left\langle\dot{u}_{n}(t)\right)+z_{n}^{*}(t) ; \dot{u}_{n}(t)\right\rangle+\left\langle-\dot{u}_{n}(t)-z_{n}^{*}(t) ; v\right\rangle \leq \delta k \mu_{n}, \text { for all } v \in C(t) .
$$

Taking the supremum on $v$ over $C(t)$ and integrating over $I$, we get

$$
\int_{0}^{T}\left\langle\dot{u}_{n}(t)+z_{n}^{*}(t) ; \dot{u}_{n}(t)\right\rangle+\delta_{C(t)}^{*}\left(-\dot{u}_{n}(t)-z_{n}^{*}(t)\right) d t \leq \delta k T \mu_{n}, \text { for any } n .
$$

Let us prove that $\dot{u}(t) \in C(t)$ a.e. on $I$. Fix any $\xi \in H$ and let $t \in I$ such that $\dot{u}(t)$ exists. Then

$$
\begin{aligned}
\langle\xi ; \dot{u}(t)\rangle & =\left\langle\xi ; \dot{u}(t)-\dot{u}_{n}(t)\right\rangle+\left\langle\xi ; \dot{u}_{n}(t)\right\rangle \\
& \leq\left\langle\xi ; \dot{u}(t)-\dot{u}_{n}(t)\right\rangle+\delta_{C\left(\theta_{n}(t)\right)}^{*}(\xi)
\end{aligned}
$$


By integrating over any measurable set $A \subset I$, we obtain

$$
\begin{aligned}
\int_{A}\langle\xi ; \dot{u}(t)\rangle d t & =\int_{A}\left\langle\xi ; \dot{u}(t)-\dot{u}_{n}(t)\right\rangle d t+\int_{A}\left\langle\xi ; \dot{u}_{n}(t)\right\rangle d t \\
& \leq \int_{A}\left\langle\xi ; \dot{u}(t)-\dot{u}_{n}(t)\right\rangle d t+\int_{A} \delta_{C\left(\theta_{n}(t)\right)}^{*}(\xi) d t .
\end{aligned}
$$

From the Hausdorff Lipschitz continuity of $C$, we deduce easily

$$
\limsup _{n} \int_{A} \delta_{C\left(\theta_{n}(t)\right)}^{*}(\xi) d t \leq \int_{A} \delta_{C(t)}^{*}(\xi) d t .
$$

Also, the weak convergence of $\dot{u}_{n}$ to $\dot{u}$ in $L^{\infty}(I, H)$ entails

$$
\lim _{n} \int_{A}\left\langle\xi ; \dot{u}(t)-\dot{u}_{n}(t)\right\rangle d t=0 .
$$

Hence, for any $\xi \in H$ and any measurable set $A \subset I$, we have

$$
\int_{A}\langle\xi ; \dot{u}(t)\rangle d t \leq \limsup _{n}\left(\int_{A}\left\langle\xi ; \dot{u}(t)-\dot{u}_{n}(t)\right\rangle d t+\int_{A} \delta_{C\left(\theta_{n}(t)\right)}^{*}(\xi) d t\right) \leq \int_{A} \delta_{C(t)}^{*}(\xi) d t .
$$

Thus, it follows that $\dot{u}(t) \in C(t)$ for a.e. $t \in I$.

Using now the weak star convergence of $-\dot{u}_{n}-z_{n}^{*}$ to $-\dot{u}-z^{*}$ in $L^{\infty}(I, H)$ and Lemma 2.4, we obtain

$$
\int_{0}^{T} \delta_{C(t)}^{*}\left(-\dot{u}(t)-z^{*}(t)\right) d t \leq \liminf _{n} \int_{0}^{T} \delta_{C(t)}^{*}\left(-\dot{u}_{n}(t)-z_{n}^{*}(t)\right) d t
$$

Using the strong convergence of $z_{n}^{*}(t)$ to $z^{*}(t):=F(t, u(t))$ for all $t \in I$, we obtain

$$
\lim _{n} \int_{0}^{T}\left\langle z_{n}^{*}(t) ; \dot{u}_{n}(t)\right\rangle d t=\int_{0}^{T}\left\langle z^{*}(t) ; \dot{u}(t)\right\rangle d t .
$$

On the other hand we have by the weak star convergence of $\dot{u}_{n}$ to $\dot{u}$ in $L^{\infty}(I, H)$

$$
\int_{0}^{T}\langle\dot{u}(t) ; \dot{u}(t)\rangle d t \leq \liminf _{n} \int_{0}^{T}\left\langle\dot{u}_{n}(t) ; \dot{u}_{n}(t)\right\rangle d t,
$$

and hence the properties of the limit inferior and the previous relations ensure

$$
\begin{aligned}
\int_{0}^{T}\left\langle\dot{u}(t)+z^{*}(t) ; \dot{u}(t)\right\rangle d t & =\int_{0}^{T}\langle\dot{u}(t) ; \dot{u}(t)\rangle d t+\int_{0}^{T}\left\langle z^{*}(t) ; \dot{u}(t)\right\rangle d t \\
& \leq \liminf _{n} \int_{0}^{T}\left\langle\dot{u}_{n}(t) ; \dot{u}_{n}(t)\right\rangle d t+\lim _{n} \int_{0}^{T}\left\langle z_{n}^{*}(t) ; \dot{u}_{n}(t)\right\rangle d t \\
& \leq \liminf _{n} \int_{0}^{T}\left\langle\dot{u}_{n}(t)+z_{n}^{*}(t) ; \dot{u}_{n}(t)\right\rangle d t .
\end{aligned}
$$

Combining this inequality with (3.10) and (3.11) we obtain

$$
\begin{aligned}
\int_{0}^{T} & {\left[\left\langle\dot{u}(t)+z^{*}(t) ; \dot{u}(t)\right\rangle+\delta_{C(t)}^{*}\left(-\dot{u}(t)-z^{*}(t)\right)\right] d t } \\
& \leq \liminf _{n} \int_{0}^{T}\left[\left\langle\dot{u}_{n}(t)+z_{n}^{*}(t) ; \dot{u}_{n}(t)\right\rangle+\delta_{C(t)}^{*}\left(-\dot{u}_{n}(t)-z_{n}^{*}(t)\right)\right] d t \\
& \leq \lim _{n} \delta k T \mu_{n}=0,
\end{aligned}
$$


and so

$$
\int_{0}^{T} \delta_{C(t)}^{*}\left(-\dot{u}(t)-z^{*}(t)\right) d t \leq \int_{0}^{T}\left\langle-\dot{u}(t)-z^{*}(t) ; \dot{u}(t)\right\rangle d t .
$$

Using the fact that $\dot{u}(t) \in C(t)$ a.e. on $I$, we obtain

$$
\int_{0}^{T} \delta_{C(t)}^{*}\left(-\dot{u}(t)-z^{*}(t)\right) d t=\int_{0}^{T}\left\langle-\dot{u}(t)-z^{*}(t) ; \dot{u}(t)\right\rangle d t,
$$

which ensures

$$
\delta_{C(t)}^{*}\left(-\dot{u}(t)-z^{*}(t)\right) d t=\left\langle-\dot{u}(t)-z^{*}(t) ; \dot{u}(t)\right\rangle, \text { a.e. on } I,
$$

and so

$$
\left\langle-\dot{u}(t)-z^{*}(t) ; \dot{u}(t)\right\rangle \geq\left\langle-\dot{u}(t)-z^{*}(t) ; v\right\rangle \text {, for all } v \in C(t) \text { and for a.e. on } I ;
$$

that is,

$$
\left\langle-\dot{u}(t)-z^{*}(t) ; v-\dot{u}(t)\right\rangle \leq 0 \text { for all } v \in C(t) \text { and for a.e. on } I .
$$

This ensures that

$$
-\dot{u}(t)-z^{*}(t) \in N(C(t), \dot{u}(t)) \text { for a.e. on } I .
$$

Thus the proof of (3.5) is complete. To achieve the proof of the theorem, we have to prove that the solution $u$ is Lipschitz. Using the construction of the sequences $u_{n}$ in the proof of Theorem 3.1, we have for the same $\lambda$ and for any $t, s \in I$ that

$$
\left\|u_{n}(t)-u_{n}(s)\right\| \leq \lambda|t-s| .
$$

Using the fact that $u_{n}(t)$ converges weakly to $u(t)$ for all $t \in I$ and the weak l.s.c. of the norm, we deduce

$$
\|u(t)-u(s)\| \leq \liminf _{n}\left[\left\|u_{n}(t)-u_{n}(s)\right\|\right] \leq \lambda|t-s| .
$$

This completes the proof of the theorem.

Taking $F \equiv 0$, we get the following existence result considered in [27], where the set-valued mapping $C$ has been taken with cone values and $0 \in C(t), \forall t \in I$, but their proof contains many gaps. Here we prove the same result with a correct proof and with weak assumptions on $C$.

Theorem 3.3. Let $X$ be a separable Hilbert space and assume that $C$ satisfies the assumptions in Theorem [3.1. Then for every $x_{0} \in X$, there exists a unique Lipschitz continuous mapping $u: I \rightarrow H$ satisfying

$$
-\dot{u}(t) \in N(C(t) ; \dot{u}(t)) \text {, a.e. on } I \text {, with } u(0)=x_{0},
$$

and

$$
\|\dot{u}(t)\| \leq R+\left\|x_{0}\right\| \text { a.e. on } I .
$$

Proof. The existence of solutions follows directly from Theorem 3.2. The proof of the uniqueness follows from the monotony of the normal cone to closed convex sets in Hilbert spaces, and to do that we follow the same ideas in [22]. Let $u_{1}$ and $u_{2}$ be two solutions of (3.12). Then by the definition of the normal cone we have for a.e. $t \in I$ that

$$
\left\langle-\dot{u}_{i}(t), v-\dot{u}_{i}(t)\right\rangle \leq 0, \text { for } i=1,2,
$$

and so by the fact that $u_{i}(t) \in C(t)$ we obtain

$$
\left\langle-\dot{u}_{1}(t), \dot{u}_{2}(t)-\dot{u}_{1}(t)\right\rangle \leq 0 \text { and }\left\langle-\dot{u}_{2}(t), \dot{u}_{1}(t)-\dot{u}_{2}(t)\right\rangle \leq 0
$$


and hence

$$
\left\|\dot{u}_{2}(t)-\dot{u}_{1}(t)\right\|^{2}=\left\langle\dot{u}_{2}(t)-\dot{u}_{1}(t), \dot{u}_{2}(t)-\dot{u}_{1}(t)\right\rangle \leq 0, \text { a.e. on } I .
$$

This ensures that $\dot{u}_{1}(t)=\dot{u}_{1}(t)$ a.e. on $I$, and so by the fact $u_{1}(0)=u_{2}(0)=x_{0}$ we obtain

$$
u_{1}(t)=u_{1}(0)+\int_{0}^{t} \dot{u}_{1}(s) d s=u_{2}(0)+\int_{0}^{t} \dot{u}_{2}(s) d s=u_{2}(t),
$$

which gives the uniqueness of the solution.

Now, we are interested by the following new type of differential inclusion in reflexive Banach spaces. Find a couple $(u, v)$ of Lipschitz mappings $u: I \rightarrow X$ and $v: I \rightarrow X$ such that $\dot{v}(t) \in C(t)$ a.e. on $I$, and

$$
\left\{\begin{array}{l}
-J(\dot{u}(t)) \in N(C(t) ; \dot{v}(t))+F(t, v(t)) \quad \text { a.e. on } I \\
u(0)=v(0)=x_{0} \in X
\end{array}\right.
$$

Theorem 3.4. Assume that the same assumptions in Theorem 3.1 are satisfied. In addition we assume that $F$ is a completely continuous mapping. Then for every $x_{0} \in X$, there exists a couple $(u, v)$ of Lipschitz solutions of (VSPP).

Proof. By Theorem 3.1, there exist sequences of mappings $\theta_{n}, \rho_{n}: I \rightarrow I, u_{n}: I \rightarrow$ $X$ such that $\left\|u_{n}(t)\right\| \leq T(R+2 \beta)+\left\|x_{0}\right\|$, and $\left\|\dot{u}_{n}(t)\right\| \leq R+2 \beta$, and for almost all $t$ in $I$,

$$
-J\left(\dot{u}_{n}(t)\right) \in N\left(C\left(\theta_{n}(t)\right) ; \dot{u}_{n}(t)\right)+F\left(\rho_{n}(t), u_{n}\left(\rho_{n}(t)\right)\right),
$$

with $u_{n}(0)=x_{0}$. Using the fact that $L^{\infty}\left(I, X^{*}\right)$ and $L^{\infty}(I, X)$ are the dual spaces of the separable Banach spaces $L^{1}(I, X)$ and $L^{1}\left(I, X^{*}\right)$, we can extract subsequences still denoted $\dot{u}_{n}$ and $J\left(\dot{u}_{n}\right)$ such that

$$
\dot{u}_{n} \rightarrow z \text { weak star in } L^{\infty}(I, X)
$$

and

$$
J\left(\dot{u}_{n}\right) \rightarrow v_{*} \text { weak star in } L^{\infty}\left(I, X^{*}\right) .
$$

Let

$$
u(t)=x_{0}+\int_{0}^{t} j^{*}\left(v_{*}(s)\right) d s, \text { where } j^{*}\left(v_{*}(s)\right) \in J^{*}\left(v_{*}(s)\right)
$$

and

$$
v(t)=x_{0}+\int_{0}^{t} z(s) d s .
$$

Clearly, we have for a.e. $t$ in $I, J(\dot{u}(t))=v_{*}(t)$ and $\dot{v}(t)=z(t)$. The Lipschitz continuity of both $u$ and $v$ follows from their definitions and the boundedness of $\left\|\dot{u}_{n}(t)\right\|$. Then for all $t \in I$,

$$
u_{n}(t)=x_{0}+\int_{0}^{t} \dot{u}_{n}(s) d s \rightarrow x_{0}+\int_{0}^{t} z(s) d s=v(t), \text { weakly in } X,
$$

and so $u_{n}\left(\rho_{n}(t)\right) \rightarrow v(t)$ weakly in $X$, for all $t \in I$. Using then the complete continuity of $F$ we obtain that $z_{n}^{*}(t):=F\left(\rho_{n}(t), u_{n}\left(\rho_{n}(t)\right)\right)$ converges strongly in $X^{*}$ to $z^{*}(t):=F(t, v(t))$, for all $t \in I$. 
Now, let us prove that $\dot{v}(t) \in C(t)$ a.e. on $I$. Let $x^{*}$ be any element in $X^{*}$, and let $t \in I$ be such that the derivative $\dot{v}(t)$ exists. Then

$$
\begin{aligned}
\left\langle x^{*}, \dot{v}(t)\right\rangle & =\left\langle x^{*}, \dot{v}(t)-\dot{u}_{n}(t)\right\rangle+\left\langle x^{*}, \dot{u}_{n}(t)\right\rangle \\
& \leq\left\langle x^{*}, \dot{v}(t)-\dot{u}_{n}(t)\right\rangle+\delta_{C\left(\theta_{n}(t)\right)}^{*}\left(x^{*}\right) .
\end{aligned}
$$

By integrating over any measurable set $A \subset I$, we obtain

$$
\begin{aligned}
\int_{A}\left\langle x^{*}, \dot{v}(t)\right\rangle d t & =\int_{A}\left\langle x^{*}, \dot{v}(t)-\dot{u}_{n}(t)\right\rangle d t+\int_{A}\left\langle x^{*}, \dot{u}_{n}(t)\right\rangle d t \\
& \leq \int_{A}\left\langle x^{*}, \dot{v}(t)-\dot{u}_{n}(t)\right\rangle d t+\int_{A} \delta_{C\left(\theta_{n}(t)\right)}^{*}\left(x^{*}\right) d t .
\end{aligned}
$$

As in the proof of Theorem 3.2 we deduce from the Hausdorff Lipschitz continuity of $C$ and from the weak star convergence of $\dot{u}_{n}$ to $\dot{u}$ in $L^{\infty}(I, X)$ that

$$
\limsup _{n}\left(\int_{A}\left\langle x^{*} ; \dot{v}(t)-\dot{u}_{n}(t)\right\rangle d t+\int_{A} \delta_{C\left(\theta_{n}(t)\right)}^{*}\left(x^{*}\right) d t\right) \leq \int_{A} \delta_{C(t)}^{*}\left(x^{*}\right) d t .
$$

Therefore, for any $x^{*} \in X^{*}$ and any measurable set $A \subset I$, we have

$$
\int_{A}\left\langle x^{*} ; \dot{v}(t)\right\rangle d t \leq \int_{A} \delta_{C(t)}^{*}\left(x^{*}\right) d t .
$$

Thus, it follows that $\dot{v}(t) \in C(t)$ for a.e. $t \in I$.

Now let us prove that the couple $(u, v)$ is a solution of our problem. By the weak star convergence of $-J\left(\dot{u}_{n}\right)-z_{n}^{*}$ to $-J(\dot{u})-z^{*}$ in $L^{\infty}\left(I, X^{*}\right)$ and Lemma 2.4 we get

$$
\int_{0}^{T} \delta_{C(t)}^{*}\left(-J(\dot{u}(t))-z^{*}(t)\right) d t \leq \liminf _{n} \int_{0}^{T} \delta_{C(t)}^{*}\left(-J\left(\dot{u}_{n}(t)\right)-z_{n}^{*}(t)\right) d t .
$$

Using the weak star convergence of $J\left(\dot{u}_{n}\right)$ to $J(\dot{u})$ in $L^{\infty}\left(I, X^{*}\right)$ and the weak star convergence of $\dot{u}_{n}$ to $\dot{v}$ in $L^{\infty}(I, X)$, we can write

$$
\lim _{n} \int_{0}^{T}\left\langle J\left(\dot{u}_{n}(t)\right), \dot{u}(t)\right\rangle d t=\int_{0}^{T}\langle J(\dot{u}(t)), \dot{u}(t)\rangle d t=\int_{0}^{T}\|\dot{u}(t)\|^{2} d t
$$

and

$$
\lim _{n} \int_{0}^{T}\left\langle J(\dot{u}(t)), \dot{u}_{n}(t)\right\rangle d t=\int_{0}^{T}\langle J(\dot{u}(t)), \dot{v}(t)\rangle d t
$$

Using the monotonicity of $J$ we write

$$
\left\langle J\left(\dot{u}_{n}(t)\right)-J(\dot{u}(t)), \dot{u}_{n}(t)-\dot{u}(t)\right\rangle \geq 0 \text {, a.e. on } I .
$$

Therefore,

$$
\begin{aligned}
\int_{0}^{T}\left\|\dot{u}_{n}(t)\right\|^{2} d t & =\int_{0}^{T}\left\langle J\left(\dot{u}_{n}(t)\right), \dot{u}_{n}(t)\right\rangle d t \\
& \geq \int_{0}^{T}\left\langle J\left(\dot{u}_{n}(t)\right), \dot{u}(t)\right\rangle d t+\int_{0}^{T}\left\langle J(\dot{u}(t)), \dot{u}_{n}(t)\right\rangle d t-\int_{0}^{T}\|\dot{u}(t)\|^{2} d t .
\end{aligned}
$$


Taking the limit inferior on both sides, we obtain that

$$
\begin{aligned}
\underset{n}{\liminf } & \int_{0}^{T}\left\|\dot{u}_{n}(t)\right\|^{2} d t \\
& \geq \int_{0}^{T}\langle J(\dot{u}(t)), \dot{u}(t)\rangle d t+\int_{0}^{T}\langle J(\dot{u}(t)), \dot{v}(t)\rangle d t-\int_{0}^{T}\|\dot{u}(t)\|^{2} d t \\
& \geq \int_{0}^{T}\langle J(\dot{u}(t)), \dot{v}(t)\rangle d t .
\end{aligned}
$$

Now we use the strong convergence of the sequence $z_{n}^{*}(t)$ to $z^{*}(t)$, for all $t \in I$, to deduce

$$
\int_{0}^{T}\left\langle z^{*}(t) ; \dot{v}(t)\right\rangle d t=\lim _{n} \int_{0}^{T}\left\langle z_{n}^{*}(t) ; \dot{u}_{n}(t)\right\rangle d t .
$$

Therefore, using this equality with the relations (3.15) and (3.16) and the properties of limit inferior we obtain

$$
\begin{aligned}
& \int_{0}^{T}\left[\delta_{C(t)}^{*}\left(-J(\dot{u}(t))-z^{*}(t)\right)+\left\langle J(\dot{u}(t))+z^{*}(t), \dot{v}(t)\right\rangle\right] d t \\
& \leq \liminf _{n} \int_{0}^{T} \delta_{C(t)}^{*}\left(-J\left(\dot{u}_{n}(t)\right)-z_{n}^{*}(t)\right) d t \\
& \quad+\liminf _{n} \int_{0}^{T}\left\langle J\left(\dot{u}_{n}(t)\right)+z_{n}^{*}(t), \dot{u}_{n}(t)\right\rangle d t \\
& \quad \leq \liminf _{n}^{T} \int_{0}^{*} \delta_{C(t)}^{*}\left(-J\left(\dot{u}_{n}(t)\right)-z_{n}^{*}(t)\right)+\left\langle J\left(\dot{u}_{n}(t)\right)+z_{n}^{*}(t), \dot{u}_{n}(t)\right\rangle d t
\end{aligned}
$$

It follows from the inclusion (3.14) and the definition of the normal cone that

$$
\left\langle-J\left(\dot{u}_{n}(t)\right)-z_{n}^{*}(t) ; w-\dot{u}_{n}(t)\right\rangle \leq 0, \quad \text { for all } w \in C\left(\theta_{n}(t)\right) \text {, a.e. on } I \text {. }
$$

Now let any $t \in I$ for which (3.18) holds and let any $v \in C(t)$. By the Hausdorff Lipschitz property of $C$, we can choose $w \in C\left(\theta_{n}(t)\right)$ such that $\|w-v\| \leq k\left|t-\theta_{n}(t)\right|$. Then we get

$$
\begin{aligned}
\left\langle-J\left(\dot{u}_{n}(t)\right)-z_{n}^{*}(t) ; v-\dot{u}_{n}(t)\right\rangle & =\left\langle-J\left(\dot{u}_{n}(t)\right)-z_{n}^{*}(t) ;\left[w-\dot{u}_{n}(t)\right]+[v-w]\right\rangle \\
& \leq\left\|J\left(\dot{u}_{n}(t)\right)+z_{n}^{*}(t)\right\|\|v-w\| \\
& \leq \delta\|v-w\| \\
& \leq \delta k \mu_{n}
\end{aligned}
$$

a.e. on $I$, with $\delta=R+3 \beta$, and so for a.e. $t \in I$ we get

(3.19) $\left\langle J\left(\dot{u}_{n}(t)\right)+z_{n}^{*}(t) ; \dot{u}_{n}(t)\right\rangle+\left\langle-J\left(\dot{u}_{n}(t)\right)-z_{n}^{*}(t) ; v\right\rangle \leq \delta k \mu_{n}$, for all $v \in C(t)$.

Taking the supremum on $v$ over $C(t)$ and integrating over $I$, we get

$$
\int_{0}^{T}\left\langle J\left(\dot{u}_{n}(t)\right)+z_{n}^{*}(t) ; \dot{u}_{n}(t)\right\rangle+\delta_{C(t)}^{*}\left(-J\left(\dot{u}_{n}(t)\right)-z_{n}^{*}(t)\right) d t \leq \delta k T \mu_{n}
$$

for any $n$. Combining the last inequality with inequality (3.17) we obtain

$$
\int_{0}^{T}\left[\delta_{C(t)}^{*}\left(-J(\dot{u}(t))-z^{*}(t)\right)+\left\langle J(\dot{u}(t))+z^{*}(t), \dot{v}(t)\right\rangle\right] d t \leq 0,
$$


and so

$$
\int_{0}^{T} \delta_{C(t)}^{*}\left(-J(\dot{u}(t))-z^{*}(t)\right) d t \leq \int_{0}^{T}\left\langle-J(\dot{u}(t))-z^{*}(t), \dot{v}(t)\right\rangle d t,
$$

which, by the fact that $\dot{v}(t) \in C(t)$, ensures that

$$
\int_{0}^{T} \delta_{C(t)}^{*}\left(-J(\dot{u}(t))-z^{*}(t)\right) d t=\int_{0}^{T}\left\langle-J(\dot{u}(t))-z^{*}(t), \dot{v}(t)\right\rangle d t .
$$

Hence for a.e. $t \in I$ we obtain

$$
\delta_{C(t)}^{*}\left(-J(\dot{u}(t))-z^{*}(t)\right)=\left\langle-J(\dot{u}(t))-z^{*}(t), \dot{v}(t)\right\rangle
$$

and hence

$$
\left\langle-J(\dot{u}(t))-z^{*}(t), w-\dot{v}(t)\right\rangle \leq 0 \text { for all } w \in C(t) .
$$

It follows then that

$$
-J(\dot{u}(t)) \in N(C(t) ; \dot{v}(t))+z^{*}(t) \subset N(C(t) ; \dot{v}(t))+F(t, u(t)) \text { a.e. on } I,
$$

and so the proof is complete.

Remark 3.2. In the general case, the relationship between $u$ and $v$ is given in terms of their derivatives as follows:

$$
\int_{0}^{T}\left\langle\dot{v}(t), x^{*}\right\rangle d t=\lim _{n} \int_{0}^{T}\left\langle\dot{w}_{n}(t), x^{*}\right\rangle d t, \text { for all } x^{*} \in X^{*}
$$

and

$$
\int_{0}^{T}\langle J(\dot{u}(t)), x\rangle d t=\lim _{n} \int_{0}^{T}\left\langle J\left(\dot{w}_{n}(t)\right), x\right\rangle d t, \text { for all } x \in X,
$$

for some sequence of mappings $w_{n}: I \rightarrow X$. So, assuming that the duality mapping $J$ on $X$ satisfies: if for any sequence $x_{n} \in L^{\infty}(I, X)$ one has

$$
\begin{aligned}
& {\left[\int_{0}^{T}\left\langle J(x(t)), x_{n}(t)-x(t)\right\rangle d t \rightarrow 0\right]} \\
& \quad \Rightarrow\left[\int_{0}^{T}\left\langle x(t), J\left(x_{n}(t)\right)-J(x(t))\right\rangle d t \rightarrow 0\right],
\end{aligned}
$$

then we obtain $u(t)=v(t)$ for all $t \in I$, and so (VSPP) has the form

$$
\left\{\begin{array}{l}
-J(\dot{u}(t)) \in N(C(t) ; \dot{u}(t))+F(t, u(t)) \quad \text { a.e. on } I, \\
u(0)=x_{0} \in X .
\end{array}\right.
$$

Clearly the assumption (A) is satisfied whenever the space $X$ is assumed to be Hilbert, and so Theorem 3.2 can be deduced as a corollary from Theorem 3.4.

We present two illustrative examples of our abstract results.

Example 3.5 (Differential Variational Inequalities (DVI)). Let $H=L^{2}(0, T, \mathbb{R})$, $g:[0, T] \times[0, T] \rightarrow[0,+\infty)$ be a Lipschitz non-increasing function w.r.t. the first variable with ratio $k>0$, and let $C(t)=\left\{z \in L^{2}(0, T, \mathbb{R}):-g(t, s) \leq z(s) \leq\right.$ $g(t, s)$, a.e. on $[0, T]\}$. Consider the following differential variation inequality: Find $u:[0, T] \rightarrow H$ such that $u(0)=x_{0} \in H$ and

(DVI) $\langle\dot{u}(t)-F(t, u(t)) ; v-\dot{u}(t)\rangle \geq 0$, for all $v \in C(t)$, a.e. on $[0, T]$,

where $F:[0, T] \times H \rightarrow H$ is a bounded completely continuous mapping. Let us prove the existence of solutions for (DVI) by using our abstract results proved in 
the previous sections. First, we rewrite (DVI) in the form of (SPP) in Theorem 3.2 Clearly $C$ has closed convex values, and so using the definition of normal cones for convex sets, (DVI) is equivalent to

$$
\begin{aligned}
\dot{u}(t)-F(t, u(t)) & \in-N(C(t) ; \dot{u}(t)) \Leftrightarrow \\
\dot{u}(t) & \in-N(C(t) ; \dot{u}(t))+F(t, u(t)) .
\end{aligned}
$$

Now, observe that for any $t_{1}, t_{2} \in[0, T]$ and for any $x \in C\left(t_{2}\right)$,

$$
d_{C\left(t_{1}\right)}(x)=\inf _{z \in C\left(t_{1}\right)}\|x-z\|_{L^{2}} \leq\left\|g\left(t_{1}, \cdot\right)-g\left(t_{2}, \cdot\right)\right\|_{L^{2}} \leq k\left|t_{2}-t_{1}\right| .
$$

So $\sup _{x \in C\left(t_{2}\right)} d_{C\left(t_{1}\right)}(x) \leq k\left|t_{2}-t_{1}\right|$. Similarly, we have $\sup _{x \in C\left(t_{1}\right)} d_{C\left(t_{2}\right)}(x) \leq$ $k\left|t_{2}-t_{1}\right|$ and hence $\mathcal{H}\left(C\left(t_{2}\right) ; C\left(t_{1}\right)\right) \leq k\left|t_{2}-t_{1}\right|$. This ensures the Hausdorff Lipschitz property of $C$ with ratio $k$. Since $0 \in C(t), \forall t \in[0, T]$, the assumption $d_{C(t)}(0)<R, \forall t \in[0, T]$ for any $R>0$, is satisfied. Then all the assumptions of Theorem 3.2 are satisfied and using (3.21), we obtain the existence of solutions for (DVI).

This example can be extended to the case of $X=L^{p}(0, T, \mathbb{R})(1<p<\infty)$ which is a separable reflexive smooth Banach space (not necessarily Hilbert).

Example 3.6 (Differential Complementarity Problems (DCP)). Let $K$ be a closed convex cone in $\mathbb{R}^{m}$ containing $0, I=[0, T]$, and let $f: I \times \mathbb{R}^{m} \times \mathbb{R}^{m} \rightarrow \mathbb{R}^{m}$ be a bounded continuous function. Find an absolutely continuous map $x: I \rightarrow \mathbb{R}^{m}$ and $u: I \rightarrow \mathbb{R}^{m}$ satisfying a.e. on $I$ the following:

$$
\begin{aligned}
\dot{x}(t) & =f(t, x(t))+u(t), \\
K^{*} \ni u(t) & \perp \dot{x}(t) \in K .
\end{aligned}
$$

First, note that a vector $u$ belongs to $-N(K ; z)$ if and only if $K^{*} \ni u \perp z \in K$. Thus (DCP) is equivalent to finding $x: I \rightarrow \mathbb{R}^{m}$ satisfying

$$
\dot{x}(t) \in-N(K ; \dot{x}(t))+f(t, x(t)) \text {, a.e. on } I .
$$

Clearly, all the assumptions of Theorem 3.2 are satisfied and thus we obtain the existence of solutions for (DCP).

Remark 3.3. Take $X=H$ and $M(t, x)=x+N(C(t) ; x)$. Then the implicit differential inclusion (3.5) becomes

$$
0 \in M(t, \dot{u}(t))+A(t, u(t)) \text {, a.e. on } I \text {. }
$$

This type of implicit differential inclusion is considered and studied in [29, 30], where $A$ and $M$ do not depend on $t$ and $H=\mathbb{R}^{n}$ and under the u.s.c. of $F$ from $\mathbb{R}^{n}$ to $\mathbb{R}^{n}$ and the monotonicity and the u.s.c. of $M$ from $\mathbb{R}^{n}$ to $\mathbb{R}^{n}$. So, we extend partially the results in [29, 30]. Also we have to mention that the author in [30] noted, "It would be interesting to see extensions of Theorem 1 [of course in [30]] to time dependent problems of the type $0 \in M(\dot{u}(t))+A(t, u(t))$ in finite and infinite dimensional spaces." In our present work we have answered his question in the affirmative.

\section{ACKNOWLEDGMENTS}

The author extends his appreciation to the Deanship of Scientific Research at King Saud University for funding the work through the research group project No. RGP-VPP-024. The author would like to thank the referee for helpful suggestions. 


\section{REFERENCES}

1. Y. Alber and I. Ryazantseva, Nonlinear ill-posed problems of monotone type. Springer, Dordrecht, 2006. MR2213033 (2007g:47109)

2. Y. Alber, Generalized Projection Operators in Banach Spaces: Properties and Applications, Funct. Different. Equations 1 (1), 1-21 (1994). MR1297965 (95i:47113)

3. Y. Alber, Metric and generalized projection operators in Banach spaces: Properties and applications, in: A. Kartsatos (Ed.), Theory and Applications of Nonlinear Operators of Monotonic and Accretive Type, Marcel Dekker, New York, 1996, pp. 15-50. MR1386667 (97b:47068)

4. J-P. Aubin, A. Cellina, Differential Inclusions: Set-Valued Maps and Viability Theory, Springer-Verlag, Berlin, 1984. MR755330 (85j:49010)

5. C. Baiocchi, A. Capelo, Variational and Quasi-variational Inequalities, Application to Free Boundary Problems, John Wiley and Sons, New York, 1984. MR.745619 (86e:49018)

6. A. Bensoussan and J.-L. Lions, Impulse Control and Quasi Variational Inequalities, GauthierVillars, Bordas, Paris, 1984. MR756234 (85f:49019)

7. M. Bounkhel, Existence and uniqueness of some variants of nonconvex sweeping Processes, J. of Nonlinear and Convex Analysis 8, No. 2 (2007), 311-323. MR2351911 (2008f:34155)

8. M. Bounkhel and L. Thibault, Nonconvex sweeping process and prox-regularity in Hilbert space, J. Nonlinear Convex Anal. 6, No. 2 (2005), 359-374. MR2159846 (2006d:49031)

9. M. Bounkhel and R. Al-yusof, First and second order convex sweeping processes in Reflexive smooth Banach spaces, Set-Valued Var. Anal. 18 (2010), no. 2, 151-182. MR2645247 (2011d:34026)

10. J.M. Borwein, H.M. Strojwas, Proximal analysis and boundaries of closed sets in Banach space, Part I: Theory, Canad. J. Math. (2) (1986), 431-452. MR833578 (87h:90258)

11. R. W. Carroll and R. E. Showalter, Singular and Degenerate Cauchy Problems, Academic Press, 1976. MR0460842 (57:834)

12. C. Castaing, Equation differentielle multivoque avec contrainte sur letat dans les espaces de Banach. Seminaire d'analyse convex 13, Montpellier, 1978. MR.521156 (80c:34067)

13. F. H. Clarke, Optimization and Nonsmooth Analysis, John Wiley \& Sons Inc., New York, 1983. MR709590 (85m:49002)

14. F.H. Clarke, Y.S. Ledyaev, R.J. Stern, P.R. Wolenski. Nonsmooth Analysis and Control Theory, Graduate Texts in Mathematics, 178, Springer-Verlag, 1998. MR1488695 (99a:49001)

15. R. Deville, G. Godefroy, V. Zizler, Smoothness and Renormings in Banach Spaces, Pitman Monographs and Surveys in Pure and Applied Mathematics, Longman Scientific \& Technical, Harlow, UK, 1993. MR1211634 (94d:46012)

16. J. Diestel, Geometry of Banach Spaces, Selected Topics, Lecture Notes in Mathematics 485, Springer-Verlag, 1975. MR0461094 (57:1079)

17. M. Fabian, P. Habala, P. Hajek, V. Montesinos Santalucía, J. Pelant, and V. Zizler, Functional analysis and infinite-dimensional geometry. CMS Books in Mathematics/Ouvrages de Mathématiques de la SMC, 8, Springer-Verlag, New York, 2001. MR.1831176 (2002f:46001)

18. A. D. Ioffe, Proximal analysis and approximate subdifferentials, J. London Math. Soc. 2 (1990), 175-192. MR.1063554 (91i:46045)

19. K. Kuttler, Non-degenerate implicit evolution inclusions, Electronic Journal of Differential Equations, Vol. 2000 (2000), No. 34, 1-20. MR.1764715 (2001e:34124)

20. J. Li, The generalized projection operator on reflexive Banach spaces and its applications, J. Math. Anal. Appl., Vol. 306 (2005), 55-71. MR2132888 (2005m:47111)

21. J.-L. Lions, Quelques Methodes de Resolution des Problemes aux limite Non linearires, Dunod, Paris, 1969. MR0259693 (41:4326)

22. P. Manchanda and A. H. Siddiqi, A Rate-Independent Evolution Quasi-Variational Inequalities and State-Dependent Sweeping Process, Advances in Nonlinear Variational Inequalities, 5 (2002), 17-18. MR.1881205 (2003f:34113)

23. M.D.P. Monteiro Marques, Differential inclusions in non-smooth mechanical problems. Shocks and dry friction, Birkhäuser, Basel-Boston-Berlin, 1993. MR 1231975 (94g:34003)

24. J. J. Moreau, Evolution problem associated with a moving convex set in a Hilbert space, J. Diff. Eqs. 26 (1977), 347-374. MR0508661 (58:22889)

25. Z. Naniewicz and P. D. Panagiotopoulos, Mathematical theory of hemivariational inequalities and applications. Monographs and Textbooks in Pure and Applied Mathematics, 188. Marcel Dekker, Inc., New York, 1995. MR 1304257 (96d:47067) 
26. J. P. Penot and R. Ratsimahalo, Characterizations of metric projections in Banach spaces and applications, Abstr. Appl. 3 (1998), no. 1-2, 85-103. MR.1700278 (2000g:46020)

27. A. H. Siddiqi and P. Manchanda, Variants of Moreau's sweeping process, Adv. Nonlinear Var. Inequal. 5 (2002), no. 1, 1-16. MR.1881204 (2003c:34103)

28. W. Takahashi, Nonlinear Functional Analysis, Yokohama Publishers, 2000. MR 1864294 (2002k:47001)

29. G. Wenzel, On a class of implicit differential inclusions, Journal of Differential Equations 63 (1986), 162-182. MR848266 (87i:34015)

30. Zou Hua Ding, On a class of implicit differential inclusions, Proceedings of the American Mathematical Society, Volume 124, Number 3, March 1996. MR.1328345 (96f:34021)

31. M. M. Vainberg, Variational methods and method of monotone operators in the theory of nonlinear equations, John Wiley, New York, 1973.

Department of Mathematics, College of Science, King Saud University, P.O. Box 2455, Riyadh 11451, Riyadh, Saudi-Arabia

E-mail address: bounkhel@ksu.edu.sa 